\title{
Pre-Processing of Integral Images for 3-D Displays
}

\author{
Amar Aggoun, Member, IEEE
}

\begin{abstract}
This paper seeks to explore a method to accurately correct geometric distortions caused during the capture of threedimensional (3-D) integral images. Such distortions are rotational and scaling errors which, if not corrected, will cause banding and moire effects on the replayed image. The method for calculating the angle of deviation in the 3-D Integral Images is based on Hough Transform. It allows detection of the angle necessary for correction of the rotational error. Experiments have been conducted on a number of 3-D integral image samples and it has been found that the proposed method produces results with accuracy of $0.05 \mathrm{deg}$.
\end{abstract}

Index Terms-Hough transform, integral imaging, three-dimensional (3-D) display, 3-D image acquisition.

\section{INTRODUCTION}

$\mathbf{R}$ EAL WORLD information, obtained by humans is three dimensional (3-D). There is growing evidence that 3-D imaging techniques will have the potential to establish a future mass-market in the fields of entertainment (television, video game) and communications (desktop video conferencing). In experimental user-trials, subjective assessments have clearly demonstrated the increased impact of 3-D pictures compared to conventional flat-picture techniques. It is reasonable, therefore, that we humans want an imaging system that produces pictures that are as natural and real as things we see and experience every day. Three-dimensional imaging and hence, 3-D television (3DTV) are very promising approaches expected to satisfy these desires [1].

Many different approaches have been adopted in attempts to realize free viewing 3-D displays [2]-[4]. Several groups [5] have demonstrated autostereoscopic 3-D displays, which work on the principle of presenting multiple images to the viewer by use of temporal or spatial multiplexing of several discrete viewpoints to the eyes. However, these autostereoscopic 3-D displays are not truly spatial displays since they exclude vertical parallax and rely upon the brain to fuse the two disparate images to create the 3-D sensation. True autostereoscopic 3-D display systems should have parallax in all directions and present images, which allow accommodation and convergence to work in unison as in normal viewing. Integral imaging is a technique that is capable of creating and encoding a true volume spatial optical model of the object scene in the form of a planar intensity distribution by using unique optical components [6]-[13]. It is akin to holography in that 3-D information recorded on a 2-D medium can be replayed as a full 3-D optical model, however, in contrast to holography, coherent light sources are not required. This conveniently allows more conventional live capture and display procedures to be adopted. With recent progress in the theory and

Manuscript received February 7, 2006; revised June 21, 2006

The author is with the Department of Engineering and Design, Brunel University, Uxbridge UB8 3PH, U.K. (e-mail: amar.aggoun@brunel.ac.uk).

Digital Object Identifier 10.1109/JDT.2006.884691 microlens manufacturing, integral imaging is becoming a practical and prospective 3-D display technology and is attracting much interest in the 3-D area.

To date, most researchers have concentrated on establishing appropriate viewing parameter characterization and improved image generation. However, there are many data processing issues that require specialist solutions unique to integral images [14]-[18]. One of these issues is to correct geometric distortion caused during the pickup process. A 3-D integral image is represented entirely by a planar intensity distribution, which may be recorded on to a photographic film for later electronic scanning and processing or directly recorded as an intensity distribution using a charge-coupled device (CCD) with a standard camera lens. A flat panel display, for example, one using liquid crystal display (LCD) technology, is used to reproduce the captured intensity modulated image, and a microlens array reintegrates the captured rays to replay the original scene in full color and with continuous parallax in all directions. This system offers the potential for stress-free viewing by more than one person. To obtain the highest possible 3-D viewing, it is essential that the structure microlens array overlaid over the LCD panel matches the pixel structure of the planar intensity distribution displayed on the LCD. This paper proposes an automatic method, which correct rotational distortions that may occur during the capture of Integral Images.

\section{Development of InTegral 3-D Imaging}

The first person who originated the term integral 3-D imaging was G. Lippmann [19] in 1908. To record an integral photograph, Lippmann used a regularly spaced array of small lenslets closely packed together in contact with a photographic emulsion. Each lenslet views the scene at a slightly different angle to its neighbor and, therefore, a scene is captured from many view points and parallax information is recorded. After processing, if the photographic transparency is reregistered with the original recording array and illuminated by diffused white light from the rear, the object will be constructed in space by the intersection of ray bundles emanating from each of the lenslets. It is the integration of the pencil beams, which renders integral imaging unique and separates it from Gaussian imaging or holography. In replay, the reconstructed image is pseudoscopic (inverted in depth). Optical and digital techniques to convert the pseudoscopic image to an orthoscopic image have been proposed [6]-[13].

An optical configuration necessary to record one-stage orthoscopic 3-D integral images has been proposed [6]-[8] and is shown in Fig. 1. This employs a pair of microlens arrays placed back to back and separated by their joint focal length, which produces spatial inversion. The arrangement allows a pseudoscopic image to be transferred such that it can straddle a separate 


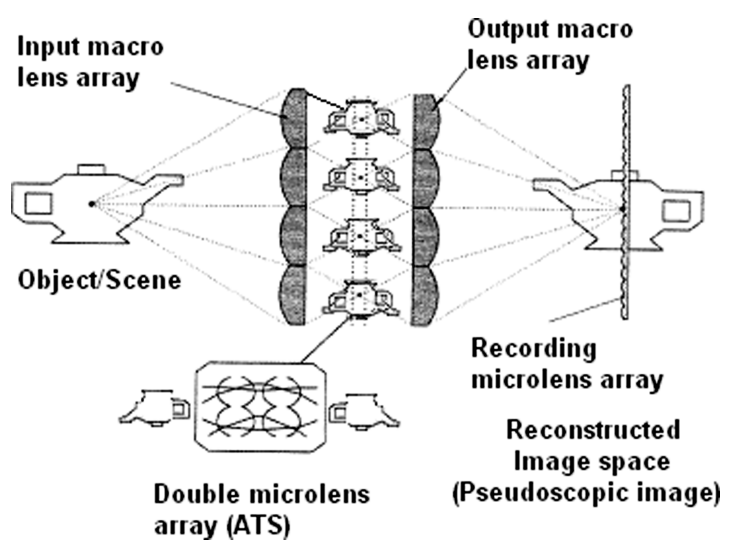

Fig. 1. An advanced integral imaging system.

microlens recording array (close imaging). The recording microlens array can be put anywhere in the transferred image space to allow the desired effect to be achieved freely: The object can be entirely inside of the display, outside of the display, or even straddling the display. The space transfer imaging scheme offers the flexibility of recording the object at a desired depth.

The system uses an additional lens array, which images the object space around the plane of the microlens combination. This arrangement has been termed a two-tier optical combination. Effectively the first macro array produces a number of pseudoscopic, laterally inverted, images around the double integral microlens screen. This image is transmitted effectively negating the sign of the input angle such that each point in object space is returned to the same position in image space. The arrangement performs pseudo-phase conjugation, i.e. transfer of volumetric data in space. The image is transmitted with equal lateral longitudinal magnification, and the relative spatial coordinates, are preserved i.e. there is no inversion in the recorded image and no scale reduction in depth.

It is possible to capture integral 3-D images electronically using a commercially available CCD array [6]-[8]. This form of capture requires a high resolution CCD together with specialized optical components to record the micro-images fields produced by precision micro-optics. The two-tier system shown in Fig. 1 has been used for the capture of the integral images used in this work. The object/scene is recorded on a film placed behind the recording microlens array through a rectangular aperture. The recorded data is then scanned using a high resolution scanner.

The aperture greatly affects the characteristics of the microimages recorded. Since each micro-image is an image of the object seen through the aperture independently, its shape and size is determined by the aperture. If the field of a micro-image is fully covered by the image, it is said to be fully-filled, otherwise it is said to be under-filled or over-filled. Under-filled microimages are caused by smaller aperture size than the correct size, and lead to decreased effective viewing zone with large part of the image area invalid when the image is replayed. Over-filled micro-images on the other hand are caused by bigger aperture size than the correct one. The overlapped micro-images lead to confusion in image reconstruction, thus degrade the replay

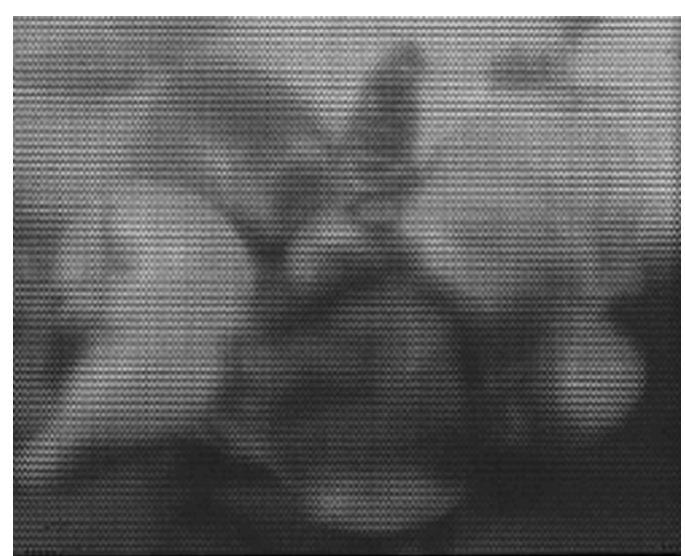

(a)

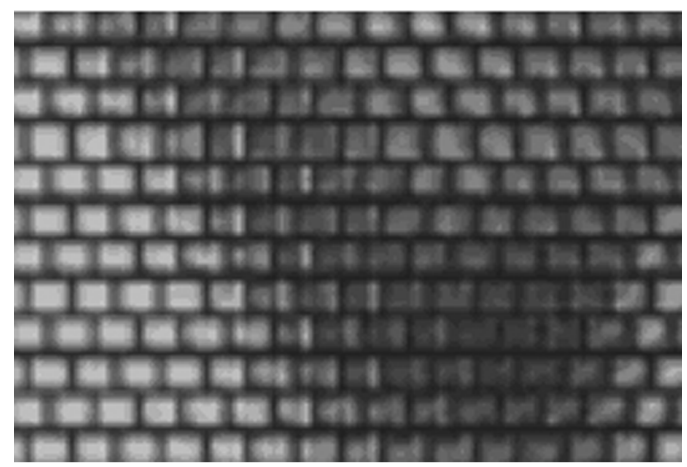

(b)

Fig. 2. (a) Example of the nature of sub-image field. (b) Magnified section.

quality. The system is setup to avoid overfilled micro-images to obtain a good 3-D integral image quality.

The system would record live images in a regular block pixel pattern. The planar intensity distribution representing an integral image is comprised of two-dimensional (2-D ) array of $M \times M$ micro-images due to the structure of the microlens array used in the capture and replay. The resulting 3-D images are termed omnidirection integral images (OII) and have parallax in all directions. The rectangular aperture at the front of the camera and the regular structure of the hexagonal microlenses array used in the hexagonal grid (recording microlens array) gives rise to a regular "brick structure" in the intensity distribution as illustrated in Fig. 2.

Unidirectional integral images (UIIs) are obtained by using a special case of the integral 3-D imaging system where one-dimensional (1-D) cylindrical microlens array is used for capture and replay instead of a 2-D array of microlenses. The resulting images contain parallax in the horizontal direction only. Fig. 3(a) shows an electronically captured unidirectional integral 3-D image and Fig. 3(b) shows a magnified section of the image. The $M$ vertically running bands present in the planar intensity distribution captured by the integral 3-D camera are due to the regular structure of the 1-D cylindrical microlens array used in the capture process.

The replay of the 3-D Integral images is achieved by placing a microlens array on the top of the recoded planar intensity distributions. The microlens array has to match exactly the structure of the planar intensity distribution. A slight mismatch would 


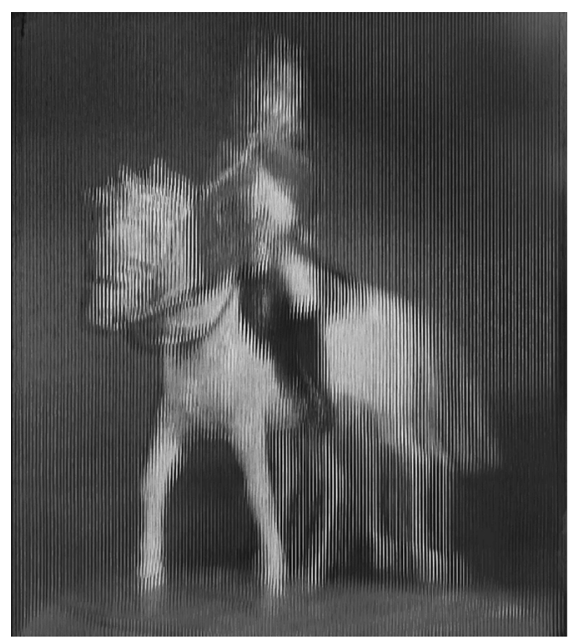

(a)

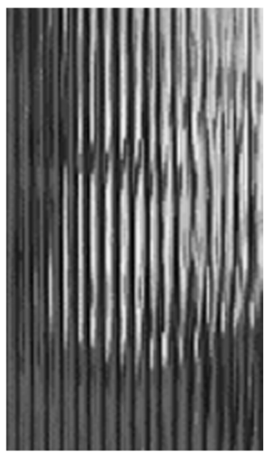

(b)

Fig. 3. An electronically captured unidirectional integral image. (a) Full. (b) Magnification.

lead to undesirable effect on the 3-D display such as banding and moiré effects. The problem of correcting the existing geometric distortions in the recorded intensity distribution implies detecting and calculating the error angle, and rotating the image with this value. The correction should be applied in such a way that the structure of the intensity distribution is preserved. An automatic method for calculating the rotational error angle is proposed based on the detection of the boundaries of the microimages in the recorded intensity distribution using the Hough Transform.

\section{ERror Angle CAlCulation IN THE UII DATA}

The process of implementing the Hough transformation on UII intensity distributions involves two steps; firstly, the boundaries of the micro-images in the captured intensity distribution data must be detected using a gradient operator [20]. The edge detection technique used is the Pythagorean sum of two Sobel gradient operators at $90 \mathrm{deg}$ each [20]. The edge-detection algorithm is followed by a standard Hough Transform to assemble the edge pixels into meaningful boundaries. The Hough transform was introduced to detect complex patterns of points in binary images and became quickly a very popular algorithm for detecting lines and simple curves.

A line detecting Hough transform is calculated by considering all of the detected edge points and representing all possible lines through these points. Considering a point $\left(x_{i}, y_{i}\right)$,

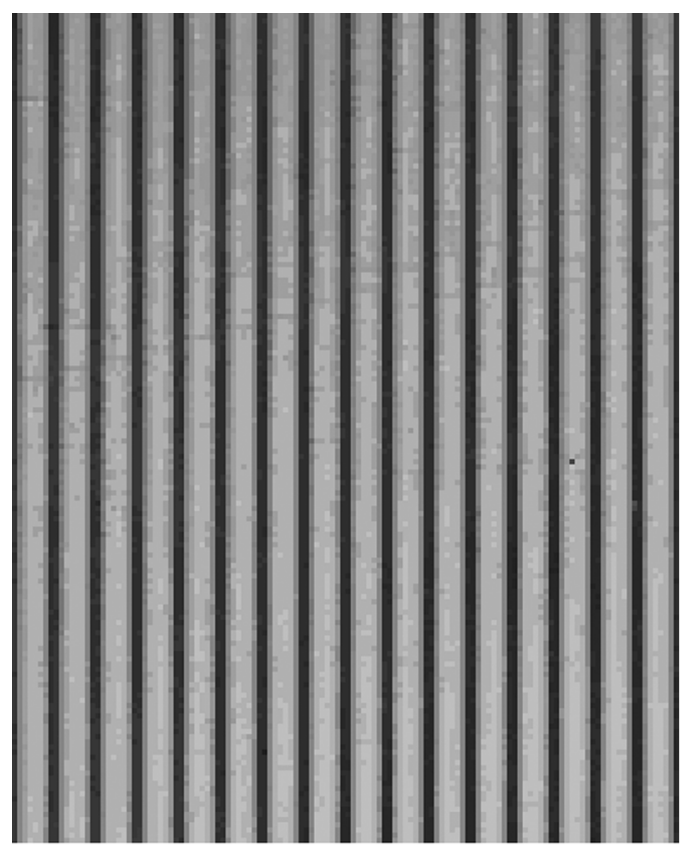

(a)

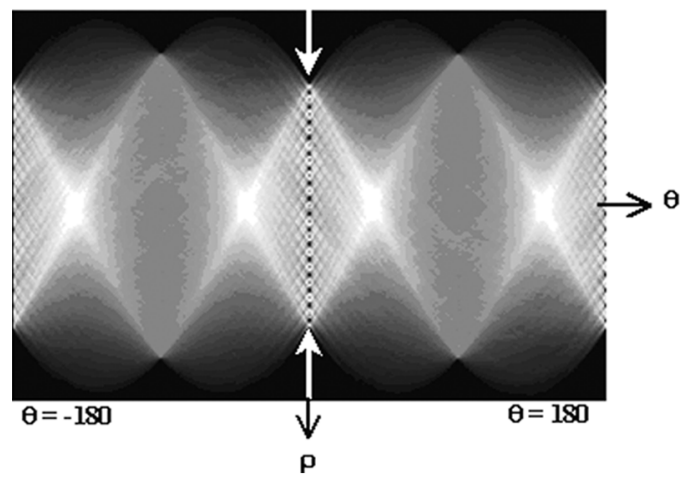

(b)

Fig. 4. (a) Perfectly aligned UII. (b) Hough image of the sample taken from a perfectly aligned UII.

the set of lines through this point is represented as the set of all possible values of $(\rho, \theta)$, where $\rho$ represents the distance between the image origin and the line, and $\theta$ the line orientation. A straight line is described by

$$
\rho=x_{i} \cos (\theta)+y_{i} \sin (\theta) .
$$

Each point $(\rho, \theta)$ in Hough space corresponds to a line at angle $\theta$ and distance $\rho$ from the origin in the original data space. The value of a function in Hough space gives the point density along a line in the data space The Hough transform is constructed by considering the sum of the sets of all lines through all edge points. The main peaks in the transform represent the boundary lines of the object. The graph is shown in rectangular coordinates $(\rho, \theta)$ for $\theta \in(-180,+180)$ in Fig. 4.

It can be observed in the Hough image represented in Fig. 4, that all intersection points are collinear (see the indication arrow) and that they form a line located at $\theta=0$ in the parameters space $(\rho, \theta)$. This observation is the starting point in our analysis. This line was termed the "Indication Line" 
(IL). The experiments were carried out with the same integral image rotated with different angles measured in respect with the horizontal axis.

The location of the $I L$ relating to the origin gives an indication of the direction of the rotation. An image rotated in a clockwise direction from the horizontal axis will produce in the Hough domain an $I L$ situated on the left side of the origin. An image rotated in an anticlockwise direction from the horizontal axis will produce in the Hough domain an $I L$ situated on the right side of the origin. The difficulty of calculating the value of $\theta$ is reduced to the estimation of the distance between the $I L$ and the origin. Since the $I L$ holds the area of interest for detecting the angle, the vicinity of this line was selected for examination. In order to determine the location of the $I L$ in the magnified Hough image, the structure of this line has been analyzed. The $I L$ is formed by many pixels with different values of grey level. However, we can assume that the $I L$ contains the largest number of black pixels in the magnified image representing the vicinity of the $I L$. To achieve a clearer representation of the $I L$, the magnified Hough image was thresholded. This results in a binary image, where the value of " 1 " was considered for each white pixel and the value of "0" was assigned to each black pixel. The sum of each column is then calculated. The maximum value is achieved for the column that contains the largest number of elements with the value of 1 . This column is then identified as the $I L$ whose location is equivalent to the value of the rotational angle.

Experiments were carried out on various samples of images with correct resolution together with the right threshold level in the process. Results showed a clear detection of the parallel boundaries of the micro-images using this process. This algorithm has been extended to OII data and results are presented and discussed in the remaining of this paper.

\section{Pre-Processing of OII Data}

The alignment process in the case OII is discussed around the position of the horizontal row of micro-images in the "brick structure," which has to produce a null angle with respect to the horizontal direction. In order to understand how the Hough transform can be applied on the captured OII intensity distribution, a test image Fig. 5(a), which simulates the structure of such set of data, is considered.

The $I L$ is located in the Hough image at $\theta=90$ as pointed out by the arrows, Fig. 5(b). A clockwise rotation (equal to $10^{\circ}$ ) was applied to the initial test image producing the image shown in Fig. 6(a). The rotation process creates a transfer of the $I L$ in the Hough image, which is denoted by $x$ [ see Fig. 6(b)].

The value of $x$ is equal to the rotational angle when the origin is considered to be at $\theta=90$. Taking into account this observation, the error angle in real OII sample data has been calculated using the Hough transform.

\section{ERror Angle Calculation In the OII Data}

To illustrate the algorithm for calculating the error angle in the captured OII data, samples have been taken from several intensity distributions including the one shown in Fig. 2(a). Due to the structure of the OII data where all the horizontal lines are parallel and uniformly distributed on the entire surface

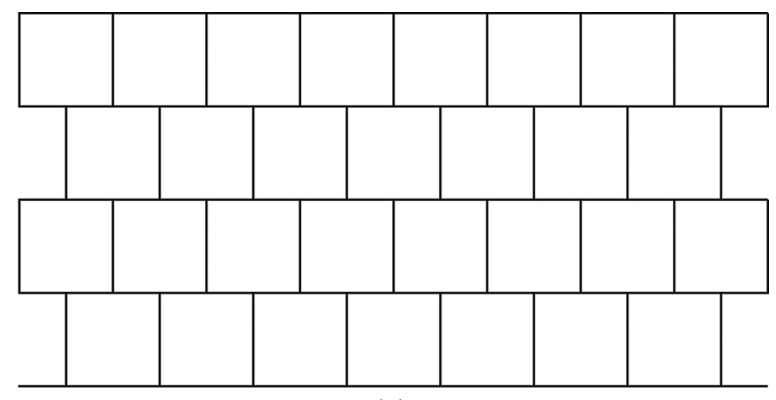

(a)

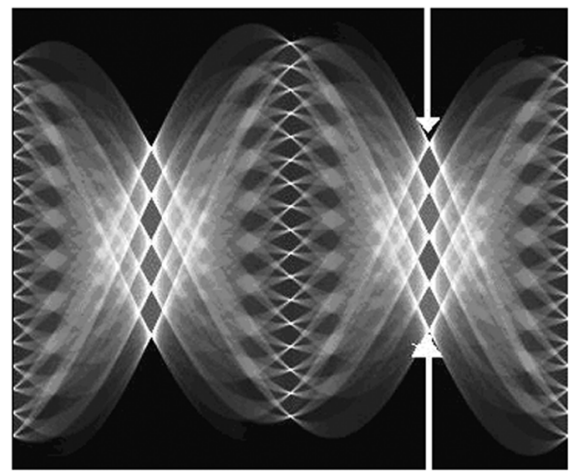

(b)

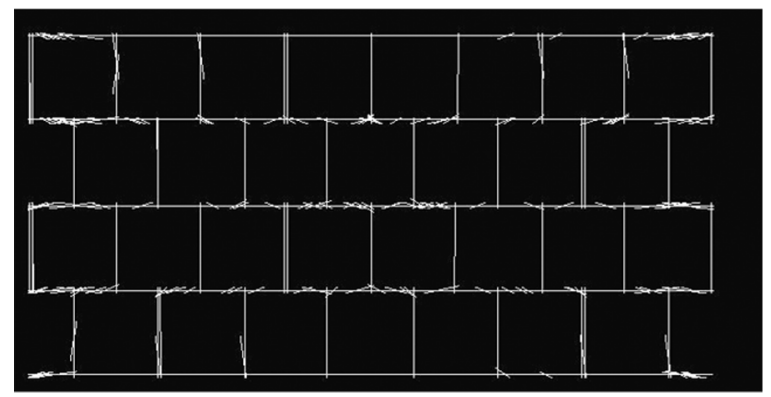

(c)

Fig. 5. Hough transform of the test image that simulates the OII structure. (a) Input image. (b) Hough representation. (c) Result produced by the Hough transform procedure.

of the image, an examination sample can be taken from any position in the image. From a visual analysis of Fig. 7 it can be seen that an anticlockwise rotation is necessary for the image in Fig. 2(a).

The method previously implemented for calculating the error angle based on the Hough transform implemented for the UII data has been applied to the captured OII intensity distribution. Because of the very small value of the rotational angle, an accurate identification of the $I L$ location was found to be difficult. For this reason a new step was added to the initial algorithm. This consists of increasing the initial rotation of the sample intensity distribution.

\section{AdDing Rotation to the SAmple Image}

In order to obtain a clear representation of the $I L$ in the Hough image of the OII sample, an extra rotation in the same direction is added to the sample image. By completing a rotation on the sample image, extra black patches have been introduced as shown in Fig. 8(a). This affects the edge detection process that 


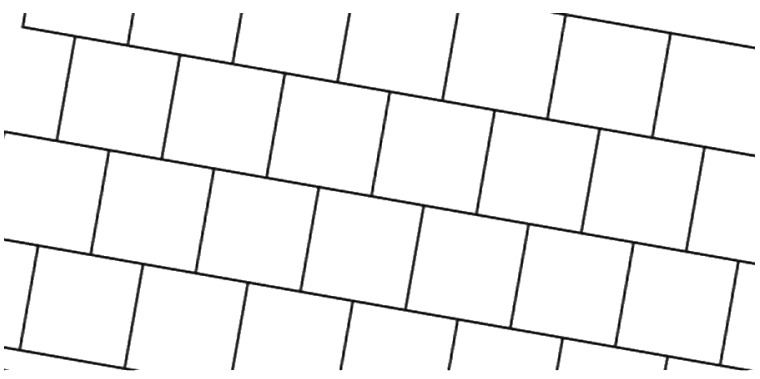

(a)

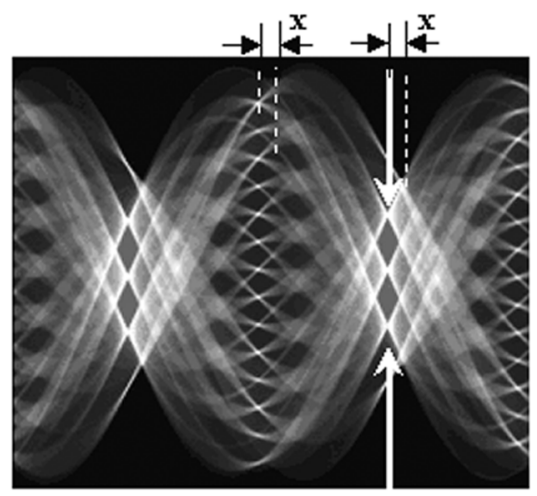

(b)

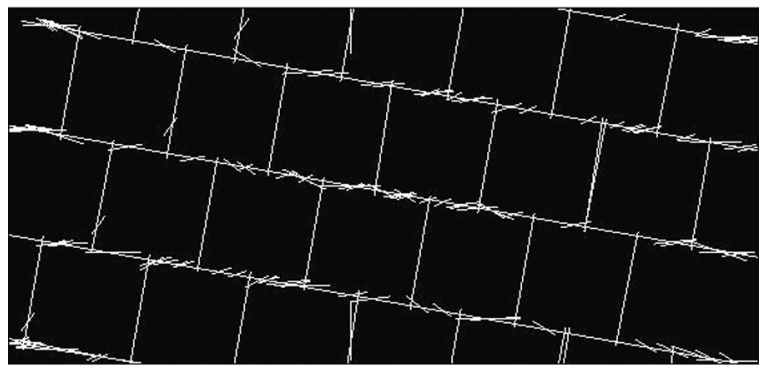

(c)

Fig. 6. Hough transform of the rotated test image. (a) Rotated input image $\left(-10^{\circ}\right)$. (b) Hough representation. (c) Result produced by the Hough transform procedure.

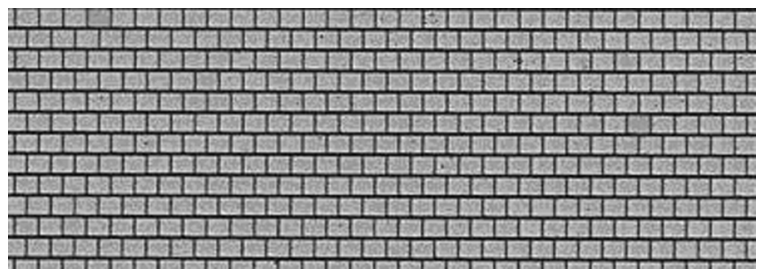

Fig. 7. Sample taken from the captured OII data shown in Fig. 2(a).

is part of the Hough transformation. To obtain a realistic result from this procedure the region of interest (ROI) was selected from the extra-rotated sample image as indicated by the dashed rectangle as illustrated in Fig. 8(b).

\section{IDENTIFICATION OF THE ROI IN THE IMAGE}

In order to identify the optimal value of the ROI, the parameters of the rectangular shape, which delimit this area, should be estimated. The initial and final values of width and height are known as well as the rotational angle. From Fig. 8(b) the fol-

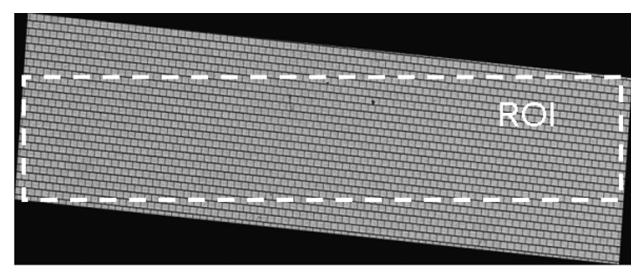

(a)

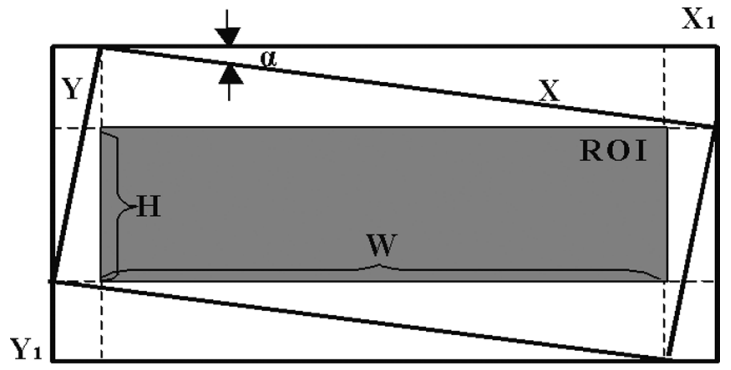

(b)

Fig. 8. (a) Example of the test sample after adding rotation. (b) Method of estimating the parameters of ROI illustrated for a clockwise rotation.

TABLE I

PARAMETERS ESTIMATION OF ROI GIVEN FOR A ClockWISE Rotation

\begin{tabular}{|l|c|}
\hline ROI parameters & Anticlockwise Rotation (+) \\
\hline Width & $W=\left(\frac{X_{1}}{\cos \alpha}-Y_{1} \sin \alpha\right)\left(1+\tan ^{2} \alpha\right)$ \\
\hline Height & $H=Y+Y_{1} \sin ^{2} \alpha-X_{1}(\sin \alpha+\tan \alpha)$ \\
\hline $\begin{array}{l}\text { Starting point } \\
\text { coordinates }\end{array}$ & $Y_{1} \sin \alpha-\tan ^{2} \alpha\left(\frac{X_{1}}{\cos \alpha}-Y_{1} \sin \alpha\right)$ \\
$X_{1} \sin \alpha$
\end{tabular}

lowing notations have been used:

$X$ width of initial image before rotation;

$Y$ height of initial image before rotation;

$\mathrm{X}_{1}$ width of the image after rotation;

$\mathrm{Y}_{1}$ height of the image after rotation;

$\alpha$ angle of clockwise rotation;

$W$ width of ROI;

$H$ height of ROI.

The width and height of the ROI area with the notation mentioned above have been calculated for clockwise rotation and are given in Table I. These values have been used in order to extract the ROI area. Similar procedure is followed to define the parameter needed to extract the ROI area for anticlockwise rotations.

After the ROI area has been identified, the Hough transform is applied on this image. The area of interest used for Hough analysis comprises the continuous horizontal and noncontinuous vertical lines consisting of average intensity pixels, which are the key element for the Hough analysis. A process of removal of small details from the image prior to object extraction and bridging the small gaps in lines is often used as a preprocessing stage to the object extraction. For this reason, a filtering stage is applied prior to application of the edge enhancement. 


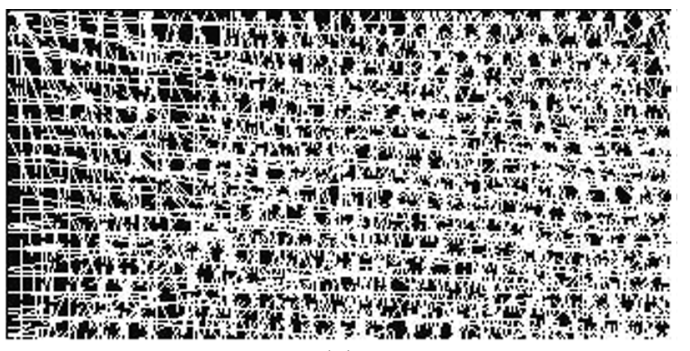

(a)

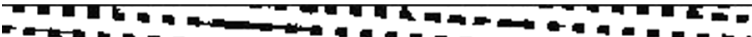
-

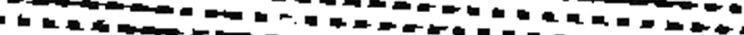
- 0 a F- = = -

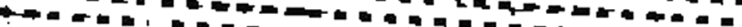
-

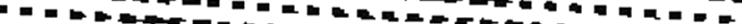

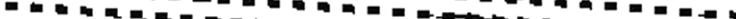

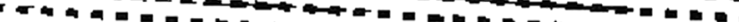

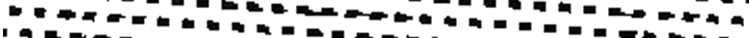
(b)

Fig. 9. Line detection images. (a) Before filtering. (b) After filtering.

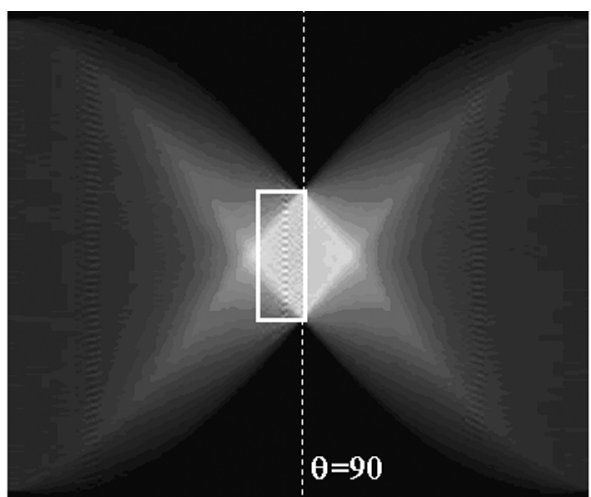

(a)

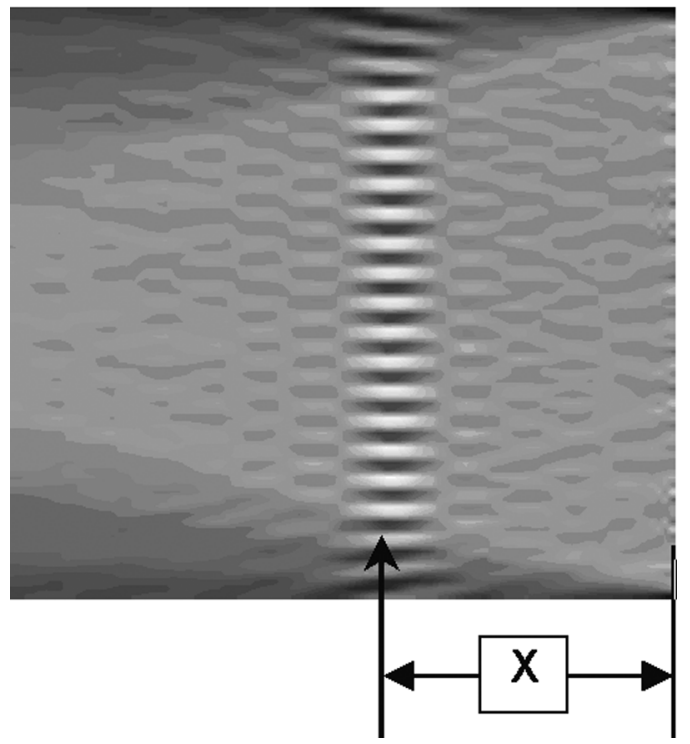

(b)

Fig. 10. (a) Hough representation for $\theta \in(0,180)$ of the test sample illustrated in Fig. 2(a). (b) The magnified section $(10 \times)$ of the IL vicinity (the square box).

A $3 \times 3$ median filter is used to achieve noise reduction while preserving edge sharpness. Application of a median filter on the

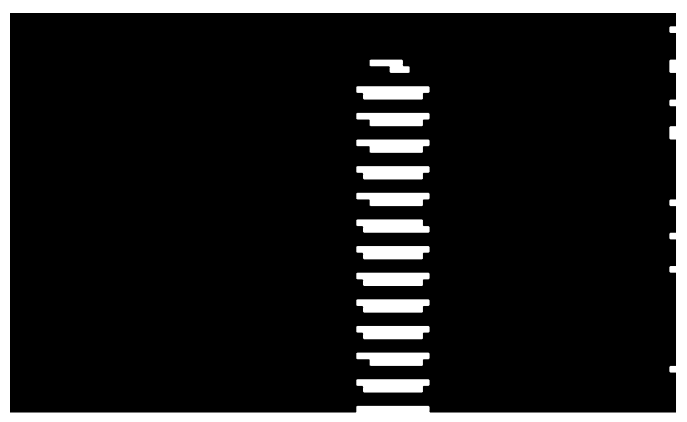

(a)

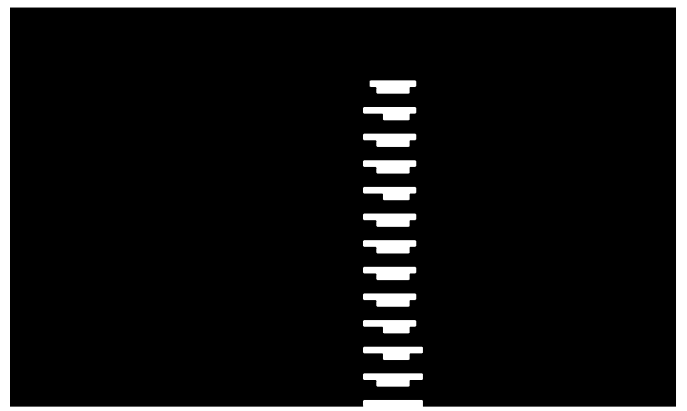

(b)

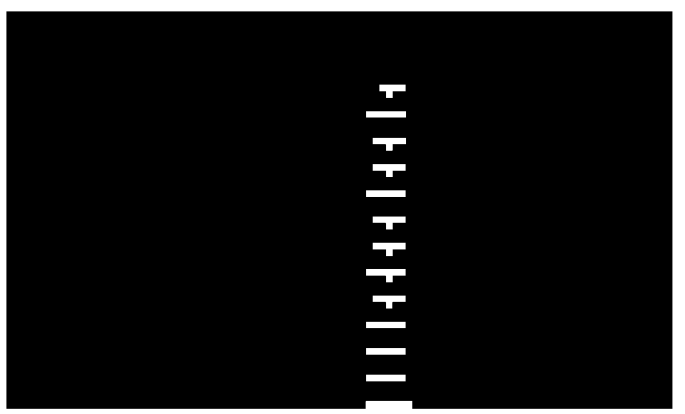

(c)

Fig. 11. Threshold image of IL vicinity of the sample image where (a) $T=180$. (b) $T=200$. (c) $T=220$.

sample images before the Hough transformation enhances the line detection image and increases the accuracy of the procedure as shown in Fig. 9.

The Hough transformation has been applied to the filtered sample images and the Hough domain representation is given in Fig. 10 for $\theta \in(0,180)$. The reference line for calculating the angle is at $\theta=90$. To facilitate the analysis of the $I L$ structure, its vicinity was selected individually for each sample image.

\section{THRESHOLDING OF THE IL VICINITY}

In order to extract the IL from the magnified section of its vicinity, a threshold process was carried out. One obvious way to identify the object from the background is to select a threshold $T$ that separates these modes.

From the histograms of the IL vicinity image, a set of threshold values are selected. The accuracy of the segmentation process which identifies the IL line is given by the value of the chosen threshold as it can be seen in Fig. 11 for the sample images in Fig. 2(a). 


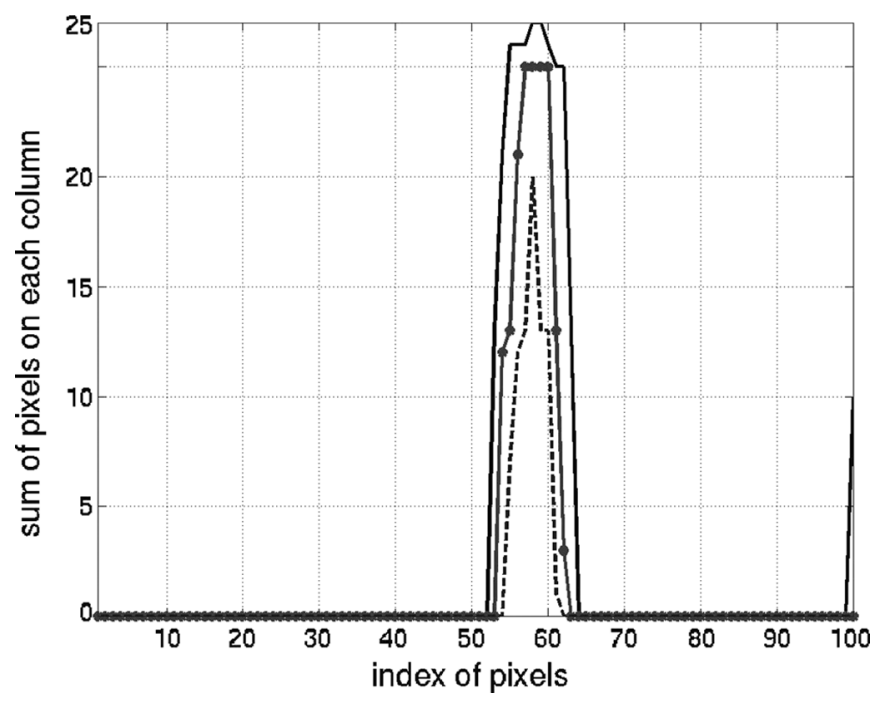

Fig. 12. Error angle representation for different threshold values.

Thus a set of binary images are formed where pixels labeled 1 correspond to objects, whereas pixels labeled 0 correspond to the background. By adding the values of the pixels on each column the graph in Fig. 12 was obtained. The maximum value in each graph gives the distance between the IL and the reference line in the Hough domain representation, which corresponds to the value of the error angle in the input data.

An accurate value of this angle is obtained by calculating the distance between the centre of the IL and the centre of the origin. The value of the angle was calculated as

$$
A_{e}=\frac{\max _{T}\left((d / m)-a_{r}\right)+\min _{T}\left((d / m)-a_{r}\right)}{2}
$$

where

$A_{e}$ error angle;

$d$ distance from max-point to reference point

$m$ magnification factor of the IL vicinity;

$a_{r}$ added rotation.

For the example described, $a_{r}=4$ and $m=10$.

The error angle values for the four test OII images calculated using this method, are $(0.575,0.225,0.435$, and 0.625$)$. Fig. 13 shows an example of the preprocessing of a UII. This UII is captured using microlens array with a pitch of $1270 \mu \mathrm{m}$ and is chosen here to clearly show the effect of preprocessing in correcting the rotational error caused during the capture.

To access the accuracy of the algorithm, a sample of UIIs which have been rotated by $10^{\circ}$ have been used as test images. The estimated angular components were found using the proposed method to be between $9.80^{\circ}$ and $9.95^{\circ}$ depending on the noise level in the captured images. Hence the proposed method could detect rotational errors with an accuracy of up to $0.05^{\circ}$.

Real-time implementations of the Hough transform for straight line detection using dedicated hardware have been reported in the literature [21]. Since the Hough transform is the main computational intensive operations of the algorithm, it is possible

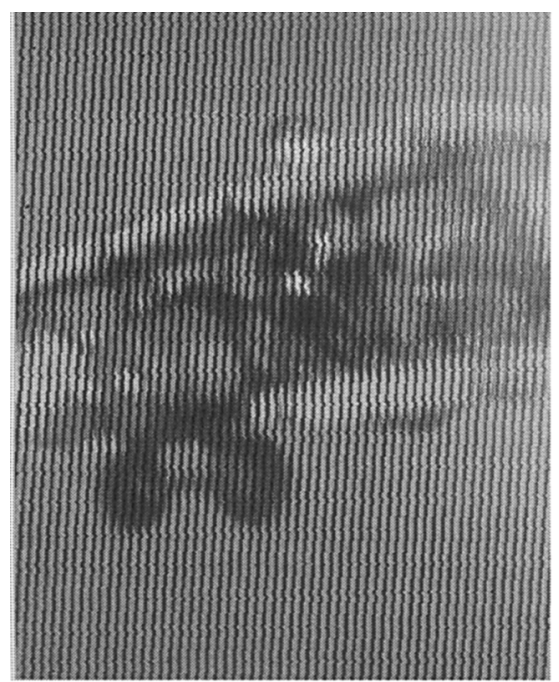

(a)

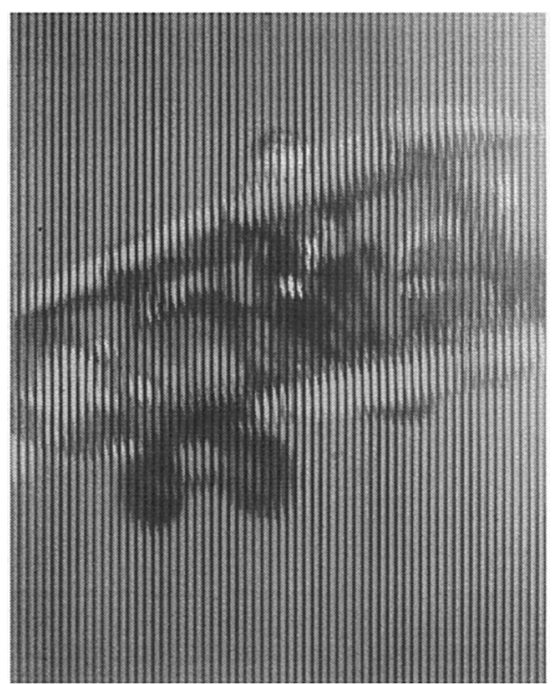

(b)

Fig. 13. Example of the preprocessing of the unidirectional integral image (a) Misaligned UII without preprocessing. (b) Perfectly aligned UII after preprocessing.

to develop a dedicated hardware for real-time operation of the proposed preprocessing technique.

\section{CONCLUSION}

This paper has covered the aspects of preparation and conversion into a proper format of 3-D integral images as a preprocess before the 3-D display. Most methods to capture integral images involves the use of a standard camera with a CCD in front a lens array, which could result in geometric distortions in the captured data. Since a microlens array is required to be placed on the recorded data to replay the 3-D Integral Imaging and a slight rotational mismatch would result in banding and moiré effect. The paper provides an automatic method using the Hough transform to detect and correct rotational error. The correct rotation adheres with the decoding lens for absolute alignment with accuracy up to 0.05 degrees. The method is easy to implement which makes it attractive for real time preprocessing of integral images on a frame by frame basis. 


\section{REFERENCES}

[1] T. Motoki, H. Isono, and I. Yuyama, "Present status of three-dimensional television research," Proc. IEEE, vol. 83, no. 7, pp. 1009-1021, Jul. 1995.

[2] T. Okoshi, Three-Dimensional Imaging Techniques. London, U.K.: Academic, 1976.

[3] S. A. Benton, Ed., Selected Papers on Three Dimensional Displays. Bellingham, WA: SPIE Optical Eng., 2001.

[4] S. V. Vladimir, J.-Y. Son, B. Javidi, S.-K. Kim, and D.-S. Kim, "Moire minimization condition in three-dimensional image displays," J. Display Technol., vol. 1, no. 2, pp. 347-353, Dec. 2005.

[5] N. A. Dodgson, "Autostereoscopic 3D displays," IEEE Computer, vol. 38 , no. 8, pp. 31-36, 2005.

[6] N. Davies et al., "Three-dimensional imaging systems: a new development," Appl. Opt., vol. 27, pp. 4520-4528, 1988.

[7] S. Monaleche, A. Aggoun, A. McCormick, N. Davies, and S. Y. Kung, "Analytical model of a $3 \mathrm{~d}$ recording camera system using circular and hexagonal based spherical microlenses," J. Opt. Soc. Amer. A, vol. 18, no. 8, pp. 1814-1821, 2001.

[8] N. Davis, M. McCormick, and M. Brewin, "Design and analysis of an image transfer system using microlens arrays," Opt. Eng., vol. 33, no. 11, pp. 3624-3633, 1994.

[9] R. Martinez-Cuenca, G. Saavedra, M. Martinez-Corral, and B. Javidi, "Extended depth-of-field 3-D display and visualization by combination of amplitude-modulated microlenses and deconvolution tools," J. Display Technol., vol. 1, no. 2, pp. 321-327, Dec. 2005.

[10] F. Okano, H. Hoshino, J. Arai, and I. Yuyama, "Real-time pickup method for a three-dimensional image based on integral photography," Appl. Opt., vol. 36, pp. 1598-1604, 1997.

[11] J. S. Jang and B. Javidi, "Time-multiplexed integral imaging," Opt. Photon. News, pp. 36-43, 2004.

[12] M. Martínez-Corral, B. Javidi, R. Martínez-Cuenca, and G. Saavedra, "Formation of real, orthoscopic integral images by smart pixel mapping," Opt. Expr., vol. 13, pp. 9175-9180, 2005.

[13] B. Javidi and F. Okano, Eds., Three-Dimensional Television, Video, and Display Technologies. New York: Springer, 2002.

[14] J. Ren, A. Aggoun, and M. McCormick, "Computer generation of integral 3D images with maximum effective viewing angle," J. Electron. Ima.g, vol. 14, 2005.
[15] C. Wu, A. Aggoun, M. McCormick, and S. Y. Kung, "3D object reconstruction from unidirectional integral images using a modified multibaseline disparity analysis," J. Electron. Imag., vol. 14, pp. ???-???, 2005.

[16] S. Manolache, S. Y. Kung, M. McCormick, and A. Aggoun, "3D-object space reconstruction from planar recorded data of 3D-integral images," J. VLSI Signal Process., vol. 35, no. 1, pp. 5-18, 2003.

[17] R. Zaharia, A. Aggoun, and M. McCormick, "Adaptive 3D-DCT compression algorithm for continuous parallax 3D integral imaging," $J$. Signal Process.: Image Commun., vol. 17, no. 3, pp. 231-242, 2002.

[18] M. C. Forman and A. Aggoun, "Quantisation strategies for 3D_DCT based compression of full parallax 3D images," presented at the IEE IPA97, 1997, Conf. Pub. No. 443, unpublished.

[19] G. Lippmann, "Eppreuves reversibles Donnat Durelief," J. Phys. Paris, vol. 821,1908 .

[20] E. R. Davies, Machine Vision, Theory, Algorithms, Practicalities, 2nd ed. New York: Academic, 1997.

[21] K. Mayasandra et al., "A distributed arithmetic hardware architecture for real-time Hough transform based segmentation," Can. J. Elect. Comput. Eng., vol. 30, no. 4, 2005.

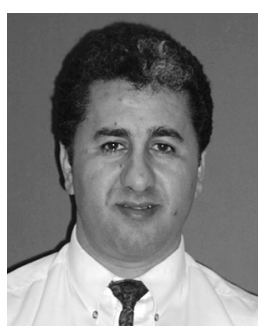

Amar Aggoun (M'99) received the Ingenieur d'Etat degree in electronic engineering from Ecole $\mathrm{Na}$ tionale Polytechnique of Algiers (ENPA), Algiers, Algeria, in 1986 and the Ph.D. degree in compressed video signal processing from Nottingham University, Nottingham, U.K., in 1991.

From 1991 to 1993 he was with Nottingham University as a research fellow in digital video signal processing. From 1993 to 2005, he was with De Montfort University, Location??, U.K., as a Principle Lecturer in Electronic Engineering. In 2005, he joined the School of Design and Engineering at Brunel University, Uxbridge, U.K., as a Reader in 3-D Imaging Technologies.

His current research Interests include computer generation and live capture of 3-D integral images, depth measurement and volumetric data reconstruction from 3-D integral images, 3-D video coding, computer vision, and real-time digital image processing architectures. 